\title{
Investigation of Cyanide Ligand as an Active Site Probe of Human Heme Oxygenase
}

Tapiwa Chiura, Piotr J. Mak*

Chemistry Department, Saint Louis University, Saint Louis, Missouri, 63103, United

States

Corresponding author: Piotr J. Mak (piotr.mak@slu.edu)

Supporting Information 
Mutation Primers

D140E forward 5'- CCC CCA GAC AGC TCA CCC AGG TAG C - 3'

D140E reverse 5' - GCT ACC TGG GTG AGC TGT CTG GGG - 3'

D140N forward 5' - GGC CCC CAG ACA GAT TAC CCA GGT AGC GG - 3'

D140N reverse -5' - CCG CTA CCT GGG TAA TCT GTC TGG GGG CC - 3'

D140A forward -5'- GGC CCC CAG ACA GAG CAC CCA GGT AGC GG - 3'

D140A reverse -5’- CCG CTA CCT GGG TGC TCT GTC TGG GGG CC - 3’ 


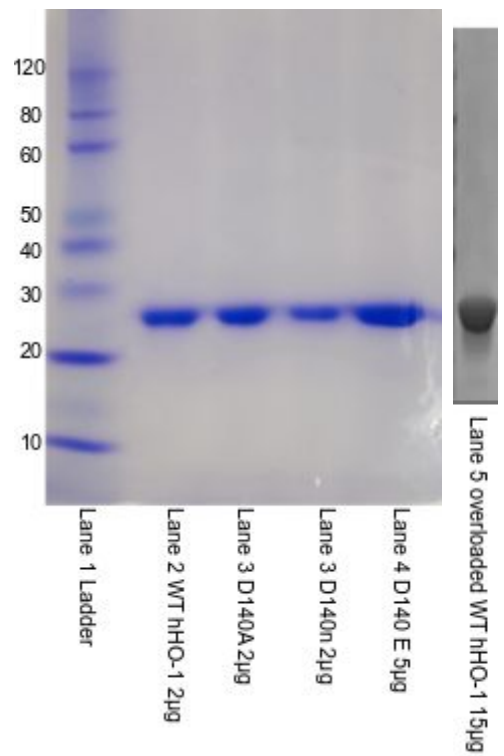

Figure S1. SDS-PAGE of hHO-1 and its D140A, D140N and D140E mutants. Lane 5 is an overloaded lane with $15 \mu \mathrm{g}$ of WT protein. 


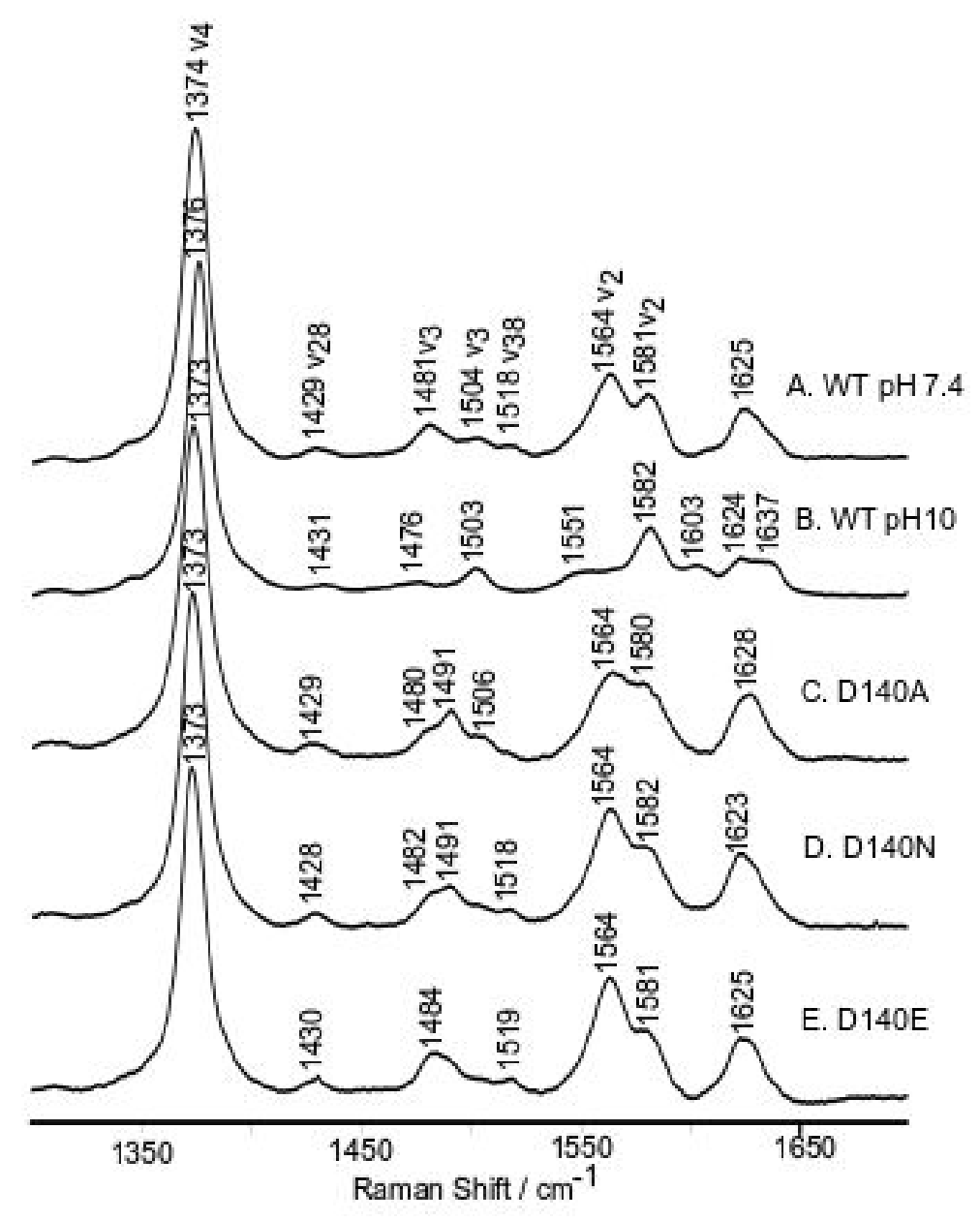

Figure S2. High frequency rR spectra of ferric WT hHO-1 at pH 7.4 (A) and pH 10 (B) as well as D140 mutants at pH 7.4, D140A (C), D140N (D) and D140E (D140E). 


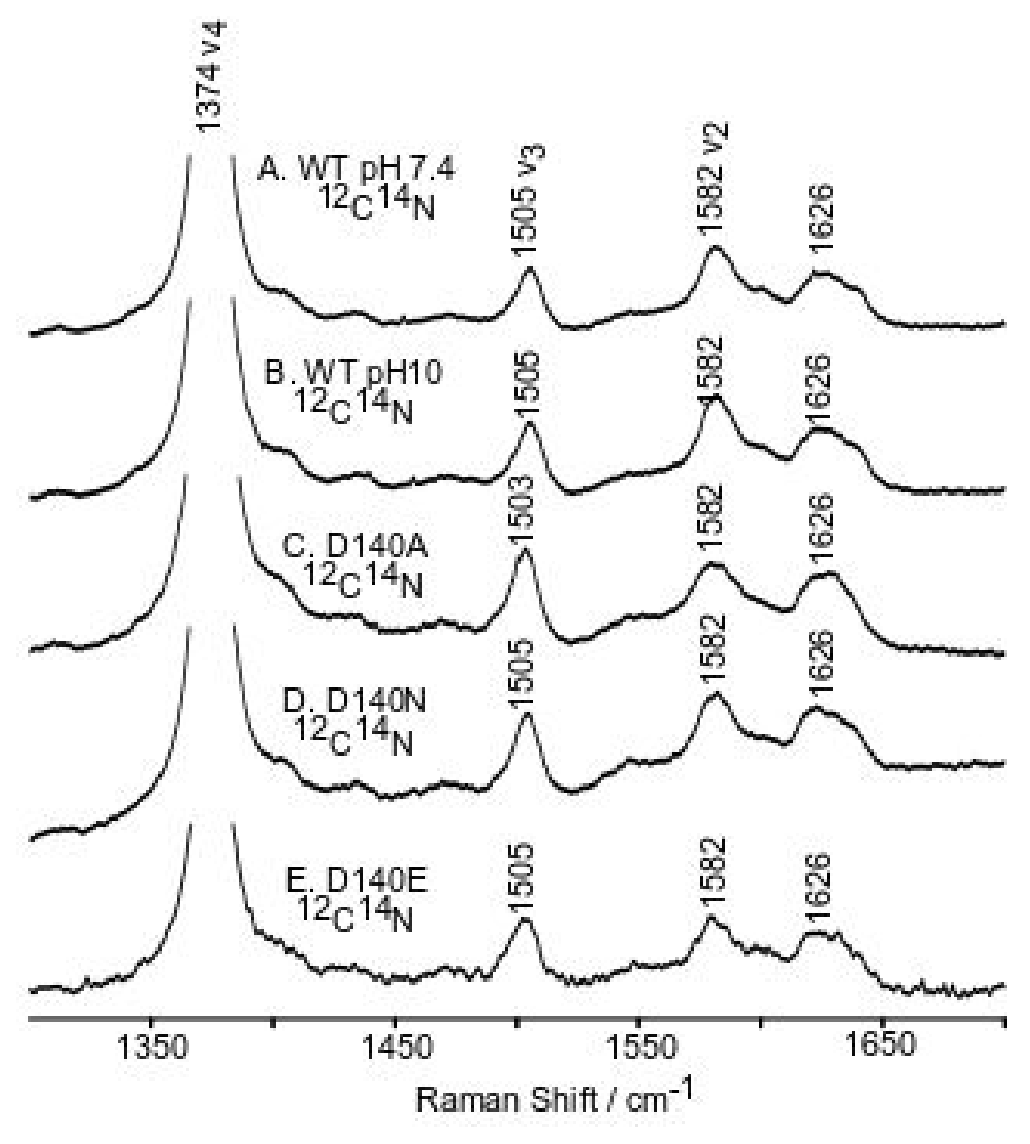

Figure S3. High frequency rR spectra of ferric ${ }^{12} \mathrm{C}^{14} \mathrm{~N}$ adducts of WT hHO-1 at pH 7.4 (A) and pH 10 (B) as well as D140 mutants at pH 7.4, D140A (C), D140N (D) and D140E (D140E). 